\title{
Sintomatologia depressiva em adolescentes iniciais - estudo de base populacional
}

\author{
Depressive symptomatology in early adolescents - population-based study
}

Luciano Dias de Mattos Souza', Rodrigo Sinnott Silva², Russélia Vanila Godoy², Ana Laura Sica Cruzeiro², Augusto Duarte Faria ${ }^{2}$, Ricardo Tavares Pinheiro ${ }^{3}$, Bernardo Lessa Horta ${ }^{4}$, Ricardo Azevedo da Silva ${ }^{5}$

\section{Palavras-chaves \\ Adolescência, saúde mental, sintomas depressivos, depressão.}

Objetivo: Verificar a prevalência de sintomatologia depressiva em adolescentes entre 11 e 15 anos em Pelotas, RS, e identificar seus fatores associados. Métodos: Estudo transversal de base populacional com amostra representativa de adolescentes da zona urbana da cidade de Pelotas, RS. Dos 448 setores censitários existentes no município, 79 foram aleatoriamente sorteados. Os jovens entre 11 e 15 anos localizados nestes setores foram convidados a responder o questionário auto-aplicado que continha perguntas sobre: idade, sexo, vida escolar, religião, atividades físicas, entretenimento, tabaco, consumo de álcool, uso de outras drogas, relacionamentos sexuais e conduta. Além disso, a sintomatologia depressiva dos adolescentes foi avaliada por meio da escala Children's Depression Inventory (CDI), também presente no instrumento de pesquisa. Para a análise dos dados foi utilizada a regressão logística ajustada ao modelo hierárquico. Resultados: $\mathrm{A}$ prevalência de depressão foi de 2,1\%. Após a regressão logística ajustada ao modelo hierárquico, estiveram significativamente associados à depressão: baixa condição socioeconômica, histórico de fracasso acadêmico, ausência de prática religiosa, abuso de álcool nos últimos 30 dias e indicativo de transtorno de conduta. Conclusão: É evidenciada a necessidade de programas preventivos e integrados de política na adolescência para depressão e comportamentos de risco à saúde.

\section{ABSTRACT}

Aim: Verify the prevalence of depressive symptomatology in adolescents aged between 11 and 15 in Pelotas, RS, and identify its associated factors. Method: A cross-sectional base-populational study with a representative sample of adolescents of urban area of Pelotas, RS. Among the 448 census tracts in the urban area of Pelotas, 79 were randomly selected. Youths between 11 and 15 years answered a confidential self-report questionnaire on issues such as: age, gender, school life, religion, physical activities, entertainment, tobacco, and alcohol consumption, use of other drugs, sexual relationship and conduct. Children's Depression Inventory (CDI) was used to quantify the depressive symptomatology. Logistic regression was used in the data analysis, which followed a

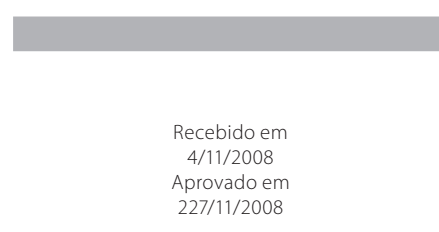

$4 / 11 / 2008$

$227 / 11 / 2008$
Trabalho realizado pelo Programa de Pós-graduação em Saúde e Comportamento da Universidade Católica de Pelotas (UCPEL). 1 Pontifícia Universidade Católica do Rio Grande do Sul (PUCRS) e Universidade Católica de Pelotas (UCPEL).

2 Universidade Católica de Pelotas (UCPEL).

3 Universidade do Porto (Portugal).

4 Mcgill University (Canadá).

5 Universidade do Minho (Portugal). 


\section{Keywords}

Adolescence, mental

health, depressive

symptoms, depression. hierarchical model. Results: Prevalence for high depressive symptomatology was 2.1\%. After a logistic regression adjusts to a hierarchical model, it was statistically significant associated to: low social economic condition, history of poor academic performance, absence of religious practice, abuse of alcohol in the previous month and indications of conduct disorders. Conclusion: It is evidenced the need of preventive and integrative policy programs in adolescence for depression and health risk behaviors.
A adolescência é um período de aquisição de autonomia caracterizado por intensas mudanças emocionais. Os sentimentos depressivos são comuns nesta etapa do desenvolvimento. No Brasil, a prevalência de elevada sintomatologia depressiva tem oscilado de $1,48 \%$ a $59,9 \%^{2}$. Muitos estudos relatam altos índices de sintomas depressivos entre adolescentes quando comparados com adultos ${ }^{3,4}$. Tais sintomas recorrentes têm sido relacionados com alta morbimortalidade ${ }^{5}$ e podem causar prejuízos em longo prazo se não-tratados ${ }^{6,7}$.

Nos estágios iniciais da adolescência, a depressão tem se mostrado associada a comportamentos de risco à saúde como o uso de álcool, tabaco e drogas ilícitas ${ }^{8,9}$. Além disso, existem indícios de que a depressão na adolescência é preditor de depressão em situações da vida adulta ${ }^{10}$. Além de danos à saúde e à qualidade de vida desses jovens, tal situação pode ser responsável pelo aumento no número de consultas de saúde pública, não se limitando aos serviços públicos de saúde mental e, assim, causando substancial impacto econômico.

Por estas razões, faz-se importante a identificação de fatores associados à elevada sintomatologia depressiva nas etapas iniciais da adolescência para auxiliar no delineamento de estratégias preventivas. Este estudo buscou identificar a prevalência de indicativo de depressão e seus fatores associados entre adolescentes com idades entre 11 e 15 anos em Pelotas, RS.

\section{MÉTODOS}

Esta pesquisa transversal de base populacional avaliou amostra representativa de adolescentes com idades entre 11 e 15 anos da zona urbana da cidade de Pelotas, RS. Os dados foram coletados em 2005.

De acordo com a estimativa de prevalência de depressão encontrada na literatura científica" ${ }^{11}$, com intervalo de confiança de $95 \%$, poder estatístico de $80 \%$ e erro amostral de 1,5\%, foi estimado o tamanho da amostra necessária para avaliação da prevalência e fatores associados da depressão na adolescência. Dessa forma, seriam necessários 655 jovens. Para controle de perdas e fatores de confusão foi acrescido 30\% do número amostral, resultando 852 adolescentes. Contudo, a presente investigação estava inserida em um projeto de maior amplitude sobre comportamentos de saúde e envolveu número de participantes maior do que o estimado.

Entre os 448 setores censitários da área urbana da cidade de Pelotas, 79 foram selecionados aleatoriamente, sendo todas as casas desses setores visitadas. Todos os adolescentes entre 11 e 15 anos identificados responderam a questionário auto-aplicado que continha questões sobre: idade, sexo, escolaridade, religião, atividades físicas, entretenimento, uso de tabaco, álcool e outras substâncias, relacionamento sexual e transtorno de conduta. O Children's Depression Inventory (CDI) foi utilizado para quantificar a sintomatologia depressiva. Aqueles jovens que apresentaram 18 ou mais pontos foram considerados com indicativo de depressão' ${ }^{12}$.

Ao chefe da família foi solicitado que respondesse determinada escala sobre características socioeconômicas e demográficas da família. O nível socioeconômico foi avaliado por meio da classificação proposta pela Associação Brasileira de Institutos de Pesquisa de Mercado (Abipeme). Esta classificação é fundamentada na acumulação de bens materiais e na escolaridade do chefe da família, sendo distribuídas as classificações nas classes A, B, C, D e E, de acordo com os escores obtidos. Por razões éticas, nenhum destes questionários foi identificado. A presente pesquisa foi aprovada pelo Comitê de Ética em Pesquisa da Universidade Federal do Rio Grande do Sul (UFRGS).

Os estudantes de psicologia foram treinados para realizar a aplicação dos questionários. Os dados coletados foram digitados no programa Epi Info versão 6.0, com a verificação automática da consistência dos dados. Para a análise dos dados, primeiro, foi calculada a prevalência da depressão, utilizando-se o referido ponto de corte para a estimativa. Após, a regressão logística foi utilizada na análise bivariada e multivariada por meio do programa SPSS 10.0 (Statistical Package for Social Science 10.0 version). A análise multivariada seguiu o modelo hierárquico, no qual cada bloco de variáveis de determinado nível foi incluído, e as variáveis com valor $p \leq 0,20$ no teste de razões de verossimilhança permaneciam no modelo. A referida análise foi controlada para efeito de conglomerado.

No modelo hierárquico, o primeiro nível incluiu: sexo, condição socioeconômica e nível educacional. O segundo nível incluiu religião, presença na escola durante o último ano e desempenho acadêmico. No terceiro nível, o uso de álcool e dro- 
gas nos últimos 30 dias foi considerado em conjunto com ter tomado porre neste período e com a iniciação sexual, e, no último nível, foi considerada a indicação transtorno de conduta.

\section{RESULTADOS}

Dos 1.265 jovens identificados pela equipe de pesquisa, $120(9,49 \%)$ se recusaram a participar ou não puderam ser entrevistados após três tentativas. A elevada prevalência da sintomatologia depressiva em adolescentes foi de 2,1\%. Na análise bivariada, as seguintes características foram associadas à elevada sintomatologia depressiva: baixa condição socioeconômica, ausência escolar no ano anterior, pobre desempenho acadêmico, não praticar religião, ter tomado porre ou ter ingerido álcool no último mês, ter tido relação sexual na vida e indicativo de conduta transtorno (Tabela 1).

Tabela 1. Descrição dos adolescentes com idades entre 11 e 15 anos na cidade de Pelotas, RS, em 2005 e análise bruta da elevada sintomatologia depressiva por meio da regressão logística (razão de chances e intervalo de confiança de 95\%).

\begin{tabular}{|c|c|c|c|c|}
\hline & $\mathrm{n}$ & $\%$ & Razão de chances (RC) - intervalo de confiança (IC) de 95\% - indicando elevada sintomatologia depressiva & p valor \\
\hline Sexo & & & & $0,185^{*}$ \\
\hline Masculino & 540 & 48,3 & Referência & \\
\hline Feminino & 577 & 51,7 & $1,94(0,73$ a 5,14$)$ & \\
\hline Condição socioeconômica & & & & $0,046^{*}$ \\
\hline Pior & 389 & 34,0 & $5,70(1,27$ a 25,63$)$ & \\
\hline Intermediária & 414 & 36,2 & $2,68(0,54$ a 13,38$)$ & \\
\hline Melhor & 342 & 29,8 & Referência & \\
\hline Idade (em anos) & & & & 0,464 \\
\hline 11 & 227 & 20,2 & Referência & \\
\hline 12 & 235 & 20,9 & $0,48(0,12$ a 1,97$)$ & \\
\hline 13 & 224 & 20,0 & $0,16(0,02$ a 1,35$)$ & \\
\hline 14 & 230 & 20,5 & $0,81(0,24$ a 2,70$)$ & \\
\hline 15 & 207 & 18,4 & $0,91(0,27$ a 3,03$)$ & \\
\hline Há quantos anos você freqüenta a escola? & & & & 0,263 \\
\hline Até 4 anos & 167 & 15,5 & $5,69(0,71$ a 45,88$)$ & \\
\hline De 5 a 7 anos & 682 & 63,1 & $4,57(0,58$ a 36,31$)$ & \\
\hline 8 ou mais anos & 231 & 21,4 & Referência & \\
\hline Você pratica alguma religiäo? & & & & $0,028^{*}$ \\
\hline Sim & 501 & 45,8 & Referência & \\
\hline Não & 594 & 54,2 & $3,45(1,15$ a 10,11$)$ & \\
\hline Você consumiu álcool no último mês? & & & & $0,005^{*}$ \\
\hline Sim & 249 & 21,7 & $3,63(1,49$ a 8,85) & \\
\hline Não & 896 & 78,3 & Referência & \\
\hline Você tomou porre no ultimo mês? & & & & $0,000^{*}$ \\
\hline Sim & 48 & 4,2 & $9,07(3,11$ a 26,45$)$ & \\
\hline Não & 1.097 & 95,8 & Referência & \\
\hline Você fumou tabaco no último mês? & & & & 0,589 \\
\hline Sim & 80 & 7,0 & $1,51(0,34$ a 6,63$)$ & \\
\hline Não & 1.065 & 93,0 & Referência & \\
\hline Você usou drogas ilícitas no ultimo mês? & & & & $0,117^{*}$ \\
\hline Sim & 39 & 3,4 & $3,34(0,74$ a 15,04$)$ & \\
\hline Não & 1,106 & 96,6 & Referência & \\
\hline Indicativo de transtorno de conduta & & & & $0,000^{*}$ \\
\hline Sim & 174 & 15,2 & $10,40(4,08$ a 26,52$)$ & \\
\hline Não & 971 & 84,8 & Referência & \\
\hline Iniciação sexual & & & & $0,003^{*}$ \\
\hline Sim & 138 & 12,1 & $4,10(1,60$ a 10,51$)$ & \\
\hline Não & 1.007 & 87,9 & Referência & \\
\hline Você pratica atividade física? & & & & 0,752 \\
\hline Fisicamente ativo & 596 & 79,7 & Referência & \\
\hline Sedentário & 152 & 20,3 & $0,78(0,17$ a 3,61$)$ & \\
\hline Vocêfreqüentou a escola no ultimo ano? & & & & $0,033^{*}$ \\
\hline Sim & 1.094 & 97,2 & Referência & \\
\hline Não & 32 & 2,8 & $5,26(1,14$ a 24,30$)$ & \\
\hline Vocêjá foi reprovado de ano na escola? & & & & $0,002^{*}$ \\
\hline Não & 588 & 56,0 & Referência & \\
\hline Uma vez & 260 & 24,8 & $8,60(1,77$ a 11,72$)$ & \\
\hline Duas vezes ou mais & 202 & 19,2 & $15,86(3,39$ a 74,19$)$ & \\
\hline
\end{tabular}


A Tabela 2 mostra que, na análise multivariada, os indivíduos com pobre desempenho acadêmico apresentaram indicativo de maior proporção de depressão. Adolescentes que não praticam religião estavam mais propensos a ter elevada sintomatologia depressiva quando comparados àqueles que praticavam qualquer religião $(O R 3,80 ; 95 \% \mid C$; 1,04 a 13,94). Aqueles indivíduos que referiram ter tomado porre nos 30 dias anteriores foram cerca de sete vezes mais sujeitos a apresentar indicativo de depressão do que aqueles que não o fizeram (OR 7,51; 95\%lC; 1,61 a 35,04). Finalmente, os indivíduos que apresentavam indicativo de transtorno de conduta relataram, em proporção 11 vezes maior, indicativo de transtorno depressivo (OR 12,18; 95\%|C; 3,72 a 39,95).

\section{DISCUSSÃO}

Esta pesquisa apresenta a prevalência de elevada sintomatologia depressiva na adolescência inicial de 2,1\%. Tal dado é semelhante ao encontrado no estudo de Patton et al." , no qual foi encontrada prevalência de depressão de 3,5\% em indivíduos entre 15 e 18 anos de idade, quando avaliados pela entrevista diagnóstica da classificação internacional de doenças e problemas relacionados à saúde (CID-10). Em estudo brasileiro com jovens entre 7 e 14 anos, Baptista e Golfeton' avaliaram a sintomatologia depressiva por meio da escala CDI. A ocorrência do indicativo de depressão foi de 1,48\% em 135 estudantes de uma escola privada de Ribeirão Preto, SP. Achado similar foi observado por Almeida Filho et al. ${ }^{3}$, em que a prevalência de depressão, em Brasília, foi de $2,8 \%$. Contudo, no Brasil, outros investigadores, utilizando a escala CDI, encontraram índices de indicativo de depressão que variam de $13,7 \%$ a 59,9\% $2,5,13$. É bastante provável que estas diferenças possam ser explicadas pelas diferenças metodológicas na estratégia de seleção da amostra, assim como por diferenças culturais dos locais onde os estudos foram conduzidos. É importante salientar que estas investigações foram realizadas exclusivamente em escolas em

Tabela 2. Modelo hierárquico final para elevada sintomatologia depressiva por meio de regressão logística (razão de chances e intervalo de confiança de 95\%). Pelotas, RS, 2005.

\begin{tabular}{|c|c|c|c|}
\hline & n e \% de elevada sintomatologia depressiva & Razão de chances (IC 95\%) - elevada sintomatologia depressiva & p valor \\
\hline \multicolumn{4}{|l|}{ Primeiro nível } \\
\hline Sexo & & & 0,200 \\
\hline Masculino & $6(31,6 \%)$ & Referência & \\
\hline Feminino & $13(68,4 \%)$ & $1,88(0,71$ a 5,02$)$ & \\
\hline Condiçăo socioeconômica & & & $0,033^{*}$ \\
\hline Pior & $12(60,0 \%)$ & $5,54(1,23$ а 24,98$)$ & \\
\hline Intermediária & $6(30,0 \%)$ & $2,11(0,41$ a 10,95$)$ & \\
\hline Melhor & $2(10,0 \%)$ & Referência & \\
\hline \multicolumn{4}{|l|}{ Segundo nível } \\
\hline Você pratica alguma religião? & & & $0,044^{*}$ \\
\hline Sim & $4(20,0 \%)$ & Referência & \\
\hline Não & $16(80,0 \%)$ & $3,80(1,04$ a 13,94$)$ & \\
\hline Você freqüentou a escola no último ano? & & & 0,060 \\
\hline Sim & $18(90,0 \%)$ & Referência & \\
\hline Não & $2(10,0 \%)$ & $5,67(0,93$ a 34,53$)$ & \\
\hline Vocêjá foi reprovado de ano na escola? & & & $0,014^{*}$ \\
\hline Não & $2(11,1 \%)$ & Referência & \\
\hline Uma vez & $7(38,9 \%)$ & $5,58(1,09$ a 28,62$)$ & \\
\hline Duas vezes ou mais & $9(50,0 \%)$ & $10,60(2,16$ a 51,91$)$ & \\
\hline \multicolumn{4}{|l|}{ Terceiro nivel } \\
\hline Você consumiu álcool no último mês? & & & 0,272 \\
\hline Sim & $10(50,0 \%)$ & $1,92(0,60$ a 6,14$)$ & \\
\hline Não & $10(50,0 \%)$ & Referência & \\
\hline Você tomou porre no ultimo mês? & & & $0,010^{*}$ \\
\hline Sim & $5(25,0 \%)$ & $7,51(1,61$ a 35,04$)$ & \\
\hline Não & $15(75,0 \%)$ & Referência & \\
\hline Você usou drogas ilícitas no último mês? & & & 0,293 \\
\hline Sim & $2(10,0 \%)$ & $2,83(0,41$ a 19,76$)$ & \\
\hline Não & $18(90,0 \%)$ & Referência & \\
\hline Iniciação sexual & & & 0,757 \\
\hline Sim & $7(35,0 \%)$ & $1,21(0,31$ a 4,93$)$ & \\
\hline Não & $13(65,0 \%)$ & Referência & \\
\hline \multicolumn{4}{|l|}{ Quarto nível } \\
\hline Indicativo de transtorno de conduta & & & $0,000^{*}$ \\
\hline $\operatorname{Sim}$ & $13(65,0 \%)$ & $12,18(3,72$ а 39,95$)$ & \\
\hline Não & $7(35,0 \%)$ & Referência & \\
\hline
\end{tabular}

* Associação estatisticamente significativa. 
diferentes regiões do país. Tal variação (0\% a 64\%) já foi descrita anteriormente na literatura científica ${ }^{14}$.

Se considerada a baixa prevalência encontrada e os comportamentos característicos da adolescência, como agressividade, alterações no sono e humor, que podem ser confundidos com manifestações clínicas, observa-se na presente investigação, bem como em outros estudos epidemiológicos, que a maioria dos jovens passa pela etapa inicial da adolescência sem distúrbios significativos, no que se refere à sintomatologia depressiva ${ }^{15}$. Isso enfatiza a idéia de que a depressão no início da adolescência não é comum e, portanto, o desenvolvimento adequado deve ser distinguido do patológico ${ }^{16}$.

Ao contrário da maioria dos estudos sobre sintomas depressivos, esta investigação não encontrou diferenças na ocorrência de depressão no que diz respeito ao sexo e à idade dos jovens. Em relação ao sexo, outras pesquisas também não encontraram diferenças entre homens e mulheres ${ }^{6,13,17}$. Contudo, parte da literatura científica sugere que as mulheres apresentam níveis mais elevados de depressão do que os homens ${ }^{2,5,11,18-21}$, sendo importante ressaltar a ausência de investigações que apresentem os homens com maior proporção de depressão do que as mulheres.

O nível socioeconômico no qual o jovem estava inserido esteve associado significativamente com a elevada sintomatologia depressiva. Kubik et al. ${ }^{17}$ encontraram maior proporção de elevada sintomatologia depressiva entre adolescentes de condição socioeconômica baixa. De maneira similar, Gorenstein et. al. ${ }^{19}$, em estudo conduzido no Brasil, observaram que os escores de depressão em jovens de escolas públicas foi maior do que adolescentes de escolas privadas, principalmente pelas diferenças socioeconômicas dos alunos. Zinn-Souza et al. ${ }^{21}$ também sugerem que problemas familiares financeiros estejam associados com sintomas depressivos em adolescentes.

Também foi observado, no presente estudo, que não praticar religião está associado com maior risco de elevada sintomatologia depressiva em jovens. Pearce, Little e Perez $^{22}$ (2003) concluíram que adolescentes que apresentaram experiências interpessoais religiosas positivas (apoio por parte da congregação ao adolescente) mostraram significativamente menos sintomas de depressão. Contudo, o referido estudo ${ }^{22}$ não encontrou associação entre o número de sintomas depressivos e a freqüência da participação do adolescente na instituição religiosa.

Neste estudo, em outro resultado oposto aos achados encontrados na literatura cientíica, a iniciação sexual não esteve associada à sintomatologia depressiva ${ }^{23,24}$. Tal incongruência pode ser explicada pela baixa prevalência de depressão encontrada, assim como pelas diferenças de faixa etária dos participantes.

No que tange à escolaridade, a sintomatologia depressiva se mostrou associada com baixo desempenho acadê- mico (já ter sido reprovado de ano) ${ }^{18}$. O viés de causalidade reversa deve ser especialmente ressaltado na interpretação de tal associação observada, uma vez que crianças com elevada sintomatologia depressiva podem estar mais propensas a serem reprovadas.

Em relação ao uso de substâncias e depressão, apenas ter tomado porre nos últimos 30 dias apresentou associação significativa com a sintomatologia depressiva. Este resultado corrobora com achados de outros estudos sobre a questão ${ }^{24,25}$. Isso serve de suporte à hipótese da automedicação, que afirma que alguns jovens têm determinadas ações em relação ao consumo de drogas (como tomar porre) para aliviar sintomas depressivos. No presente estudo, o uso de tabaco e o consumo de drogas ilícitas não apresentaram associação significativa com a elevada sintomatologia depressiva, o que contradiz a literatura científica ${ }^{17,20,21}$.

A proporção dos jovens que apresentaram elevada sintomatologia depressiva foi 12 vezes maior naqueles adolescentes que apresentaram indicativo de transtorno de conduta. Comorbidades costumam ocorrer em transtornos depressivos na adolescência; o transtorno de conduta ocorre em 15\% dos $\operatorname{casos}^{26}$. Mirza e Michael${ }^{27}$ apontam que o transtorno de conduta é a segunda comorbidade mais freqüente em adolescentes com depressão. Por outro lado, Costello et al. $.^{28} \mathrm{ob}-$ servaram que a comorbidade entre transtorno de conduta e depressão ocorre mais freqüentemente em meninas.

Sobre as limitações deste estudo, é importante ser enfatizado que o instrumento utilizado na avaliação da sintomatologia depressiva não permite o diagnóstico de depressão. Da mesma forma, o delineamento transversal não permite o estabelecimento de associações de causa e efeito. Contudo, os dados descritos nesta investigação se referem à amostra representativa de base populacional, o que limita a ocorrência de viés de seleção da amostra. A utilização de questionário auto-aplicado assegurou a confidencialidade dos dados aos participantes, minimizando eventuais erros de informação sobre os comportamentos de saúde descritos no instrumento.

Em suma, o presente estudo identificou que a elevada sintomatologia depressiva na cidade de Pelotas, RS, está associada à baixa condição socioeconômica, ao baixo desempenho acadêmico, à ausência de prática religiosa, ter tomado porre e indicativo de transtorno de conduta.

\section{CONCLUSÃO}

Estes dados sugerem que a elevada sintomatologia depressiva não possui alta prevalência na adolescência inicial, assim como ressaltam a existência de possíveis comorbidades deste quadro com comportamentos de risco à saúde. Portanto, é evidenciada a necessidade de programas de tratamento preventivo e integrado na adolescência inicial para depressão e comportamentos de risco à saúde. 


\section{CONFLITO DE INTERESSES}

A presente pesquisa foi financiada pelo Conselho Nacional de Desenvolvimento Científico e Tecnológico (CNPq), sob Processo ำ 452269/2003-2.

\section{REFERÊNCIAS}

1. Baptista CA, Golfeton JH. Prevalência de depressão em escolares de 7 a 14 anos. Rev Psiq Clin. 2000;27:253-5.

2. Jatobá JAVN, Bastos 0. Depressão e ansiedade em adolescentes de escolas públicas e privadas. J Bras Psiquiatr. 2007:56(3):171-9.

3. Almeida-Filho N, Mari JJ, Coutinho E, França JF, Fernandes J, Andreoli SB, et al. Brazilian multicentric study of psychiatric morbidity methodological features and prevalence estimates. Br J Psychiatry. 1997;171:524-9.

4. Hammen C, Brennan PA, Keenan-Miller D, Herr NR. Early onset recurrent subtype of adolescent depression: clinical and psychosocial correlates. Journal of Child Psychology and Psychiatry and Allied Disciplines. 2008;49(4):433-40.

5. Bahls SC. Depressive symptoms in adolescents of a public school. Rev Bras Psiquiatr. 2002;24(2):63-7.

6. Birmaher B, Ryan N, Williamson D, Brent DA, Kaufman J, Dahl RE, et al. Childhood and adolescent depression: a review of the past 10 years. Part I. J Am Acad Child Adolesc Psychiatry. 1996;35(11):1427-39

7. Kashner TM, Henley SS, Golden RM, Rush AJ, Jarrett RB. Assessing the preventive effects of cognitive therapy following relief of depression: a methodological innovation. J Affect Disord. 2007;104(1-3):251-61.

8. Sandí-Esquivel L, Díaz-Alvarado A. Sintomas de rebeldia, depresión y aislamiento asociados al consumo de drogas en adolescentes costarricences. Acta Pediatrica Costarricense. 1995:9(3):99-102

9. Stoduto G, Dill P, Mann RE, Wells-Parker E, Toneatto T, Shuggi R. Examining the link between drinking-driving and depressed mood. J Stud Alcohol Drugs. 2008:69(5):777-80.

10. Stein MB, Fuetsch M, Muller N, Hofler M, Lieb R, Wittchen HU. Social anxiety disorder and the risk of depression: a prospective community study of adolescents and young adults. Arch Gen Psychiatry. 2001;58(3):251-6.

11. Patton GC, Coffey C, Posterino M, Carlin JB, Wolfe R. Adolescent depressive disorder: a population based study of ICD-10 symptoms. Aust N Z J Psychiatry. 2000;34:741-7.

12. 12. Gouveia RV, Barbosa GA, Almeida HJF, Gaião AA. Inventário de depressão infantil - CDI: estudo de adaptação com escolares de João Pessoa. J Bras Psiquiatr. 1995:44(7):345-9.
13. Barbosa GA, Dias MR, Gaião AA, Lorenzo WCG. Depressão infantil: um estudo de prevalência com o CDI. Infanto. Rev Neuropsiquiatria da Infância e Adolescência. 1996;14(3):36-40.

14. Espeleta L. Epidemiologia de la depression infantil en el Municipio de Barcelona. Rev Psiquiatria da Faculdade de Medicina de Barcelona. 1998;15:255-68.

15. Offer D, Schonert-Reichl KA. Debunking the myths of adolescence: findings from recent research. J Am Acad Child Adolesc Psychiatry. 1992;31:1003-14.

16. Haarasilta L. Major depressive episode in adolescence and young adults - a nationwide epidemiological survey among 15-24 years old. Academic Dissertation (Department of Psychiatry, Medical Faculty). Helsinki: University of Helsinki [acesso em 2006 Oct 15]. Disponível em: https://0a.doria.fi/bitstream/handle/10024/37680/D803.pdf? sequence=1.

17. Kubik MY, Lytle LA, Birnbaum AS, Murray DM, Perry CL. Prevalence and correlates of depressive symptons in young adolescents. Am J Health Behav. 2003;27(5):546-53.

18. Dell'Áglio DD, Hutz CS. Depressão e desempenho escolar em crianças e adolescentes institucionalizados. Psicologia: Reflexão e Crítica. 2004;17(3):351-7.

19. Gorenstein C, Andrade L, Zanolo E, Artes R. Expression of depressive symptoms in a nonclinical brazilian adolescent sample. Can J Psychiatry. 2005;50:129-37.

20. Saluja G, lachan R, Scheidt C, Overpeck MD, Sun W, Giedd JN. Prevalence of and risk fators for depressive symptoms among young adolescents. Arch Pediatr Adolesc Med. 2004;158:760-5.

21. Zinn-Souza LC, Nagai R, Teixeira LR, Latorre MRDO, Roberts R, Cooper SP, et al. Depression in high school students. Rev Saude Publica. 2008;42(1):34-40.

22. Pearce MJ, Little TD, Perez JE. Religiousness and depressive symptoms among adolescents. J Clin Child Adolescent Psychology. 2003;32(2):267-76.

23. Dishman RK, Hales DP, Pfeiffer KA, Felton GA, Saunders R, Ward DS, et al. Physical selfconcept and self-esteem mediate cross-sectional relations of physical activity and sport participation with depression symptoms among adolescent girls. Health Psychol. 2006;25(3):396-407.

24. Perera B, Torabi MR, Jayawardana G, Pallethanna N. Depressive symptoms among adolescents in Sri Lanka: prevalence and behavioral correlates. J Adolesc Health. 2006:39(1):144-6

25. Best D, Manning V, Gossop M, Gross S, Strang J. Excessive drinking and other problem behaviours among 14-16 year old schoolchildren. Addict Behav. 2006;31(8):1424-35.

26. Soares K, Almeida-Filho N, Coutinho OESF, Mari JJ. Sintomas depressivos entre os adolescentes e adultos de uma amostra populacional de três centros urbanos brasileiros: análise dos dados do estudo multicêntrico de morbidade psiquiátrica. Rev Psiq Clin. $1999 ; 26(5): 218-24$

27. Mirza KAH, Michael A. Major depression in children and adolescents. Br J Hosp Med (Lond). 1996:55(1-2):57-61.

28. Costello EJ, Mustillo S, Erkanli A, Keeler G, Angold A. Prevalence and development of psychiatric disorders in childhood and adolescence. Arch Gen Psychiatry. 2003;60(8):837-44. 